\title{
The effects of aerobic exercise on metabolic risk, insulin sensitivity and intrahepatic lipid in healthy older people from the Hertfordshire Cohort Study: a randomised controlled trial
}

\author{
F. M. Finucane • S. J. Sharp • L. R. Purslow • K. Horton • J. Horton • D. B. Savage • \\ S. Brage • H. Besson • E. De Lucia Rolfe • A. Sleigh • H. J. Martin • A. Aihie Sayer • \\ C. Cooper • U. Ekelund • S. J. Griffin • N. J. Wareham
}

Received: 11 August 2009 /Accepted: 30 November 2009/Published online: 6 January 2010

(C) Springer-Verlag 2009

\begin{abstract}
Aims/hypothesis We sought to determine the effect of an aerobic exercise intervention on clustered metabolic risk and related outcomes in healthy older adults in a singlecentre, explanatory randomised controlled trial.

Methods Participants from the Hertfordshire Cohort Study (born 1931-1939) were randomly assigned to 36 supervised $1 \mathrm{~h}$ sessions on a cycle ergometer over 12 weeks or to a non-intervention control group. Randomisation and group allocation were conducted by the study co-ordinator, using a software programme. Those with prevalent diabetes, unstable ischaemic heart disease or poor mobility were
\end{abstract}

Electronic supplementary material The online version of this article (doi:10.1007/s00125-009-1641-z) contains supplementary material, which is available to authorised users.

F. M. Finucane $(\bowtie) \cdot$ S. J. Sharp $\cdot$ L. R. Purslow $\cdot$ K. Horton $\cdot$

J. Horton $\cdot$ S. Brage $\cdot$ H. Besson $\cdot$ E. De Lucia Rolfe $\cdot$

U. Ekelund $\cdot$ S. J. Griffin · N. J. Wareham

MRC Epidemiology Unit, Institute of Metabolic Science,

Addenbrooke's Hospital,

Box 285, Hills Road,

Cambridge CB2 0QQ, UK

e-mail: francis.finucane@mrc-epid.cam.ac.uk

D. B. Savage

Metabolic Research Laboratories, Institute of Metabolic Science, Cambridge, UK

A. Sleigh

Wolfson Brain Imaging Centre, University of Cambridge,

Cambridge, UK

H. J. Martin · A. Aihie Sayer · C. Cooper

MRC Epidemiology Resource Centre,

University of Southampton,

Southampton, UK excluded. All data were collected at our clinical research facility in Cambridge. Components of the metabolic syndrome were used to derive a standardised composite metabolic risk score (zMS) as the primary outcome. Trial status: closed to follow-up.

Results We randomised 100 participants (50 to the intervention, 50 to the control group). Mean age was 71.4 (range 67.4-76.3) years. Overall, $96 \%$ of participants attended for follow-up measures. There were no serious adverse events. Using an intention-to-treat analysis, we saw a nonsignificant reduction in $\mathrm{zMS}$ in the exercise group compared with controls $(0.07$ [95\% CI $-0.03,0.17]$, $p=0.19$ ). However, the exercise group had significantly decreased weight, waist circumference and intrahepatic lipid, with increased aerobic fitness and a $68 \%$ reduction in prevalence of abnormal glucose metabolism (OR 0.32 [95\% CI 0.11-0.92], $p=0.035)$ compared with controls. Results were similar in per-protocol analyses.

Conclusions/interpretation Enrolment in a supervised aerobic exercise intervention led to weight loss, increased fitness and improvements in some but not all metabolic outcomes. In appropriately screened older individuals, such interventions appear to be safe.

Trial registration: Controlled-trials.com ISRCTN60986572 Funding: Medical Research Council

Keywords Aerobic exercise - Hertfordshire Cohort Study Insulin sensitivity · Intrahepatic lipid · Metabolic risk ·

Randomised controlled trial
Abbreviations
DEXA Dual energy X-ray absorptiometry
HOMA-2S HOMA-derived insulin sensitivity 
IHL Intrahepatic lipid

OGIS Oral glucose insulin sensitivity

$W_{\max } \quad$ Maximum power output (predicted)

zMS Composite metabolic risk score

\section{Introduction}

Several studies have shown that individuals who are fitter and more physically active have lower mortality rates and less cardiovascular disease [1, 2]. Increases in physical activity over time are associated with lower metabolic risk and mortality [3]. Intervention trials to increase physical activity and fitness in older people tend to be restricted to specific high-risk subgroups, such as those with abnormal glucose metabolism or established cardiovascular disease. Lifestyle interventions reduce progression to diabetes in individuals with impaired glucose tolerance and the benefits of such interventions persist for several years $[4,5]$. Even in older people with established vascular disease, exercise interventions reduce cardiovascular and all-cause morbidity and mortality rates, and improve quality of life $[6,7]$. However, evidence that healthy older people derive similar benefits from exercise interventions is limited. In practice, old age is often an exclusion criterion for such studies. Notwithstanding this, public health initiatives encourage people to undertake at least moderate-intensity physical activity several times per week, irrespective of age or disease risk [8,9]. While such encouragement seems reasonable given our current knowledge of the net benefits of physical activity, the evidence base for aerobic exercise interventions in healthy older people is weak.

In the diabetes prevention trials, older participants derived more benefit from lifestyle interventions than younger ones $[5,10]$. Conversely, individuals with healthier metabolic profiles tended to derive less benefit from exercise [11]. We sought to determine the effects of aerobic exercise in healthy older people. Specifically, we hypothesised that enrolment in a fully supervised 12 week aerobic exercise intervention would lead to changes in a composite measure of metabolic risk and other clinically relevant outcomes, compared with non-intervention control participants.

\section{Methods}

Study design and setting The study rationale and design have been described in detail previously [12]. Briefly, in this single-centre, explanatory, randomised controlled trial, participants were allocated to the exercise or control group using the minimisation method, with the minimisation factors being birthweight, percentage body fat, sex and whether or not a muscle biopsy had been performed at baseline. All participants provided written, informed consent. The duration of follow-up was 12 weeks from study entry. All study measures were conducted at the Institute of Metabolic Science in Cambridge, UK, between 17 January 2007 and 15 February 2008. The research assistants taking the measurements were unaware of the participant's group allocation. The aerobic exercise intervention was delivered at a gymnasium in Hitchin, Hertfordshire. The study protocol was approved by the Hertfordshire Research Ethics Committee (Local Research Ethics Committee ref. 05/Q0201/23).

Study population We planned to recruit 100 participants from the Hertfordshire Cohort Study, a unique data resource consisting of men and women born between 1931 and 1939 and still residing in Hertfordshire, of whom almost 3,000 have been extensively characterised [13]. Specifically, potential study participants meeting inclusion criteria, living near Hitchin and deemed potentially suitable for participation by their general practitioner were identified. Those with known diabetes, untreated or unstable ischaemic heart disease or any medical condition that would prevent them from cycling unaided for at least $30 \mathrm{~min}$ were excluded from the trial. Invitations were dispatched on a phased basis until the recruitment target was achieved. In order to recruit 100 participants, we approached 591 individuals from the cohort. Recruits attended the clinical research facility after an overnight fast. Those with claustrophobia, cardiac pacemakers or metal implants were precluded from magnetic resonance scanning. Of 106 who attended the screening visit, six were deemed to be unsuitable for the study because of poor mobility, prevalent diabetes, symptoms or signs suggestive of untreated ischaemic heart disease or a combination of these factors; these six were excluded.

Measurements All measurements were undertaken by trained staff adhering to standard operating procedures. Weight was measured on a Tanita (Tokyo, Japan) scale and height with a Seca (Hamburg, Germany) wall-mounted stadiometer. Waist and hip circumferences were measured using a D-loop non-stretch fibreglass tape measure. The waist was defined as the midpoint between the lower costal margin and the level of the superior iliac crests. The hip measurement was taken at the level of the greater trochanter. Blood pressure was measured with an oscillometric device (Omron, Kyoto, Japan) using the right arm, after participants were seated quietly for $5 \mathrm{~min}$. Three measures were recorded at $1 \mathrm{~min}$ intervals and the lowest used for analyses. A resting 12-lead ECG was obtained. A dual energy X-ray absorptiometry (DEXA) scan (Lunar Prodigy Advanced; GE Healthcare, Waukesha, WI, USA) 
was used to measure bone mineral content, and lean and fat mass [14]. Magnetic resonance measures of intrahepatic lipid (IHL), and visceral and subcutaneous adipose tissue were conducted on a whole-body scanner (3T Tim Trio; Siemens, Munich, Germany), as described in detail previously [12]. A questionnaire was used to record details of current medications, smoking and alcohol use, and self-perceived functional status using the SF-8 (QualityMetric, Lincoln, RI, USA) [15]. Additionally, the average self-reported daily number of main meals, light meals and snacks over the previous 4 weeks was recorded for each participant.

Blood samples and oral glucose tolerance test A standard $75 \mathrm{~g}$ OGTT was performed. Fasting samples were taken for glucose, insulin, C-peptide and lipid profiles. After ingestion of glucose, further samples were taken every $30 \mathrm{~min}$ over $2 \mathrm{~h}$. Samples for glucose and lipid profiles were processed immediately, while those for insulin and C-peptide were spun and frozen for subsequent batch analysis. All blood samples were analysed at the Cambridge University Hospitals NHS Foundation Trust Department of Clinical Biochemistry and the NIHR Cambridge Biomedical Research Centre, Core Biochemical Assay Laboratory (CBAL). HOMA-derived insulin sensitivity (HOMA-2S) [16] was used to estimate basal insulin sensitivity, while the oral glucose insulin sensitivity (OGIS) model [17] was used to determine 'stimulated' sensitivity based on dynamic insulin responses during the OGTT.

Aerobic fitness A sub-maximal exercise test was performed on a cycle ergometer, with a starting workload of $50 \mathrm{~W}$, increasing by $10 \mathrm{~W}$ every min until $90 \%$ of the maximum agepredicted heart rate was achieved or the participant wanted to stop. An estimate of maximal aerobic fitness was obtained by extrapolating a regression line between the observed heart rate and the obtained maximum power output (predicted) $\left(W_{\max }\right)$ up to the age-predicted maximal heart rate [18].

Exercise intervention Participants attended the exercise facility for three $1 \mathrm{~h}$ sessions per week over 12 weeks, on Monday, Wednesday and Friday mornings or afternoons. All sessions were fully supervised by the exercise facilitator, and incorporated a warm-up and cool-down period. Participants used upright or recumbent cycle ergometers to achieve an exercise intensity of 50,60 and $70 \%$ of $W_{\max }$ during weeks 1 to 4,5 to 8 and 9 to 12 of the intervention, respectively. To ensure that the appropriate intensity was maintained during the sessions, heart rate was monitored (Polar F4 monitors; Bodycare Products, Southam, UK) and recorded at $5 \mathrm{~min}$ intervals. For participants on beta-blockers, the Borg scale [19] was used to assess the intensity of exercise. For those randomised to the exercise intervention, a follow-up visit to the testing facility was scheduled for precisely 2 days after completion of the final exercise session. Participants randomised to the control group were asked to continue with their usual levels of physical activity until their follow-up visit 12 weeks later.

Statistical methods The primary outcome was a composite metabolic risk score (zMS) measured at follow-up. This was derived by standardising and then summing the following variables: (systolic blood pressure+diastolic blood pressure)/2, log 2-h plasma glucose, log fasting insulin, inverted fasting HDL-cholesterol, log triacylglycerol and waist circumference, as previously described [20]. The component variables were standardised using sexspecific means and standard deviations from the larger Hertfordshire Cohort Study population, from which these participants were recruited, excluding those with prevalent diagnosed diabetes. There were several secondary outcome variables, including functional status, body composition, aerobic fitness, blood pressure, IHL, lipid profiles, and OGTT-derived measures of glucose homeostasis and glucose tolerance status.

For the primary trial efficacy analysis, the intention-totreat population included all participants who were randomised. The per-protocol analysis included exercise group participants who completed $85 \%$ or more of their prescribed exercise sessions and all the control group. The primary analysis compared the zMS at follow-up, adjusted for baseline, between the exercise and control groups, using linear regression with follow-up risk score as the outcome and baseline risk score as a covariate. This is equivalent to comparing within-person changes from baseline in each measure (with adjustment for baseline) between the exercise and control groups. Similar methods were used for the secondary outcomes. Outcomes that were not normally distributed were log-transformed prior to analysis.

With 50 individuals randomised to each group, the study had $85 \%$ power to detect a difference in mean zMS risk score of 0.6 standard deviation units between groups at follow-up, with the assumption that the risk score at followup in the control group would have a mean of 0 and a standard deviation of 1 . This estimate of effect size is consistent with our observations in a similar populationbased cohort [1]. Missing values for outcome variables at follow-up were excluded from the analysis of that variable, while for missing baseline values, the missing indicator method was used in sensitivity analyses [21].

\section{Results}

A total of 100 individuals (the target sample size) attended their screening visit and were deemed to be suitable for study participation. Their mean age was 71.4 (range 67.4- 
76.3) years; $44 \%$ were women. Baseline metabolic and anthropometric characteristics are summarised in Table 1. A family history of diabetes was present in $25 \%$ of participants, $36 \%$ were ex-smokers, $2 \%$ were current smokers and $84 \%$ drank alcohol. Statins were being taken by $20 \%$, and $15 \%$ had been prescribed beta-blockers. Overall, the groups were well matched for all characteristics at baseline. The prevalence of abnormal glucose metabolism (incorporating incident asymptomatic diabetes [five cases], impaired fasting glucose, impaired glucose tolerance or a combination of these) was $42 \%$ in exercisers and $38 \%$ in controls at baseline.

Of the 50 participants randomised to each group, 48 attended their follow-up visit 12 weeks later. Two participants from each group dropped out for reasons unrelated to the study. Figure 1 summarises study recruitment and retention. Among participants in the exercise group, 37 (74\%) attended $\geq 85 \%$ of their prescribed sessions and $20 \%$ attended all sessions. Five individuals attended less than one third of their prescribed sessions, but returned for follow-up measures. Of these, three never started the intervention, one stopped after 4 weeks due to exacerbation of pre-existing osteoarthritis in both knees and one stopped after 2 weeks because of exercise-induced palpitations, although subsequent cardiac investigations in this participant were normal.

Primary outcome Compared with the control group, there was a trend to reduced zMS in the exercise group at followup, but this did not reach statistical significance (Table 1, Fig. 2). The only component of zMS that significantly improved with exercise was waist circumference.

Table 1 Participant characteristics at baseline and follow-up

\begin{tabular}{|c|c|c|c|c|c|c|c|c|c|c|}
\hline \multirow{2}{*}{$\begin{array}{l}\text { Characteristic } \\
\text { Weight }(\mathrm{kg})\end{array}$} & \multicolumn{2}{|c|}{ Control baseline } & \multicolumn{2}{|c|}{ Control follow-up } & \multicolumn{2}{|c|}{ Exercise baseline } & \multicolumn{2}{|c|}{ Exercise follow-up } & \multirow{2}{*}{$\frac{\text { Difference }(95 \% \mathrm{CI})}{-0.83(-1.43,-0.24)}$} & \multirow{2}{*}{$\frac{p \text { value }}{0.007}$} \\
\hline & 77.3 & 14.1 & 77.2 & 14.2 & 77.2 & 16.1 & 77.0 & 16.2 & & \\
\hline Height (cm) & 169.2 & 9.4 & 169.3 & 9.6 & 167.4 & 8.0 & 167.6 & 8.0 & $-0.03(-0.28,0.22)$ & 0.80 \\
\hline BMI $\left(\mathrm{kg} / \mathrm{m}^{2}\right)$ & 26.9 & 3.6 & 26.8 & 3.6 & 27.4 & 4.9 & 27.3 & 4.8 & $-0.26(-0.50,-0.02)$ & 0.032 \\
\hline DEXA lean mass (kg) & 48.4 & 10.1 & 48.2 & 10.4 & 47.8 & 9.2 & 48.0 & 9.2 & $-0.16(-0.66,0.33)$ & 0.52 \\
\hline DEXA fat mass (kg) & 25.7 & 7.1 & 25.6 & 7.0 & 25.9 & 10.6 & 25.9 & 10.3 & $-0.001(-0.67,0.67)$ & 1.00 \\
\hline DEXA body fat (\%) & 33.3 & 7.0 & 33.4 & 7.1 & 33.2 & 8.6 & 33.1 & 8.5 & $0.08(-0.67,0.84)$ & 0.83 \\
\hline DEXA android fat mass $(\mathrm{kg})$ & 2.7 & 1.0 & 2.6 & 1.0 & 2.7 & 1.2 & 2.7 & 1.2 & $-0.06(-0.1,0.03)$ & 0.21 \\
\hline DEXA gynoid fat mass $(\mathrm{kg})$ & 4.4 & 1.2 & 4.4 & 1.3 & 4.3 & 1.7 & 4.3 & 1.7 & $-0.002(-0.1,0.1)$ & 0.96 \\
\hline Waist circumference $(\mathrm{cm})$ & 96.7 & 12.0 & 96.9 & 11.0 & 98.6 & 14.2 & 97.8 & 13.8 & $-1.7(-3.1,-0.24)$ & 0.023 \\
\hline Systolic BP (mmHg) & 134.3 & 17.3 & 131.9 & 18.9 & 138.8 & 15.0 & 135.8 & 12.5 & $1.0(-4.2,6.3)$ & 0.70 \\
\hline Diastolic BP (mmHg) & 73.4 & 8.9 & 72.4 & 9.4 & 76.8 & 8.6 & 75.2 & 9.1 & $0.9(-2.1,3.9)$ & 0.57 \\
\hline Resting pulse (bpm) & 65.2 & 8.8 & 64.4 & 10.7 & 66.0 & 9.7 & 62.1 & 7.3 & $-2.7(-5.5,0.2)$ & 0.066 \\
\hline Fitness $\left(W_{\max }\right)$ & 158.8 & 61.1 & 160.6 & 53.8 & 143.9 & 48.9 & 167.7 & 49.7 & $15.3(3.4,27.2)$ & 0.012 \\
\hline Total cholesterol (mmol/1) & 5.4 & 1.2 & 5.4 & 1.2 & 5.7 & 1.0 & 5.6 & 1.0 & $-0.009(-0.2,0.2)$ & 0.94 \\
\hline HDL-cholesterol (mmol/l) & 1.5 & 0.5 & 1.5 & 0.4 & 1.5 & 0.3 & 1.5 & 0.4 & $0.01(-0.04,0.07)$ & 0.63 \\
\hline LDL-cholesterol (mmol/l) & 3.3 & 1.0 & 3.3 & 1.0 & 3.7 & 0.8 & 3.6 & 0.8 & $-0.04(-0.2,0.2)$ & 0.70 \\
\hline Total:HDL cholesterol ratio & 3.9 & 1.2 & 3.8 & 1.2 & 4.0 & 1.0 & 3.9 & 0.9 & $-0.009(-0.2,0.2)$ & 0.92 \\
\hline Triacylglycerol $(\mathrm{mmol} / \mathrm{l})^{\mathrm{a}}$ & 1.1 & $0.8,1.6$ & 1.0 & $0.8,1.5$ & 1.2 & $0.8,1.6$ & 1.2 & $0.8,1.7$ & $1.1(0.9,1.2)$ & 0.39 \\
\hline $\operatorname{IHL}(\%)^{\mathrm{a}}$ & 3.6 & $1.4,8.3$ & 3.5 & $1.0,12.6$ & 3.7 & $1.8,10.2$ & 2.4 & $1.0,6.6$ & $0.78(0.64,0.97)$ & 0.024 \\
\hline Glucose $0 \min (\mathrm{mmol} / \mathrm{l})^{\mathrm{a}}$ & 5.0 & $4.6,5.3$ & 4.9 & $4.5,5.4$ & 4.9 & $4.6,5.4$ & 4.9 & $4.6,5.2$ & $0.99(0.96,1.02)$ & 0.62 \\
\hline Glucose $120 \min (\mathrm{mmol} / \mathrm{l})^{\mathrm{a}}$ & 7.0 & $5.5,8.6$ & 6.9 & $5.4,8.1$ & 6.9 & $5.9,8.4$ & 6.4 & $5.2,7.2$ & $0.93(0.85,1.01)$ & 0.095 \\
\hline $\mathrm{HbA}_{1 \mathrm{c}}(\%)$ & 5.7 & 0.4 & 5.7 & 0.4 & 5.7 & 0.3 & 5.7 & 0.3 & $-0.04(-0.1,0.04)$ & 0.33 \\
\hline Insulin $0 \min (\mathrm{pmol} / \mathrm{l})^{\mathrm{a}}$ & 51.9 & $44.7,81.2$ & 49.0 & $35.4,80.5$ & 51.3 & $38.1,86.3$ & 41.8 & $33.1,67.5$ & $0.91(0.78,1.06)$ & 0.210 \\
\hline Insulin $120 \min (\mathrm{pmol} / 1)^{\mathrm{a}}$ & 277.5 & 188,523 & 300.0 & 212,475 & 344.0 & $215.5,549$ & 270.0 & $149.5,421$ & $0.76(0.62,0.93)$ & 0.009 \\
\hline HOMA-2 S $(\%)^{\mathrm{a}}$ & 102.4 & $67.8,122$ & 110.5 & $73.1,149$ & 105.7 & $63.2,139$ & 128.4 & $78,165.2$ & $1.1(0.9,1.3)$ & 0.230 \\
\hline OGIS (ml $\left.\min ^{-1} \mathrm{~m}^{-2}\right)$ & 409.6 & 67.8 & 419.2 & 72.6 & 410.1 & 62.2 & 427.0 & 60.9 & $8.04(-10.1,26.2)$ & 0.38 \\
\hline zMS & -0.11 & 0.62 & -0.16 & 0.61 & -0.06 & 0.61 & -0.22 & 0.55 & $-0.07(-0.17,0.03)$ & 0.19 \\
\hline
\end{tabular}

Unless indicated, values are means and standard deviations

Difference represents the difference in within-person change in mean levels of each outcome from baseline to follow-up, adjusted for baseline ${ }^{a}$ Skewed distributions, where medians and interquartile ranges are presented, and difference is a ratio of geometric means bpm, beats per $\min$ 
Fig. 1 CONSORT flow diagram for study recruitment. IHD, ischaemic heart disease

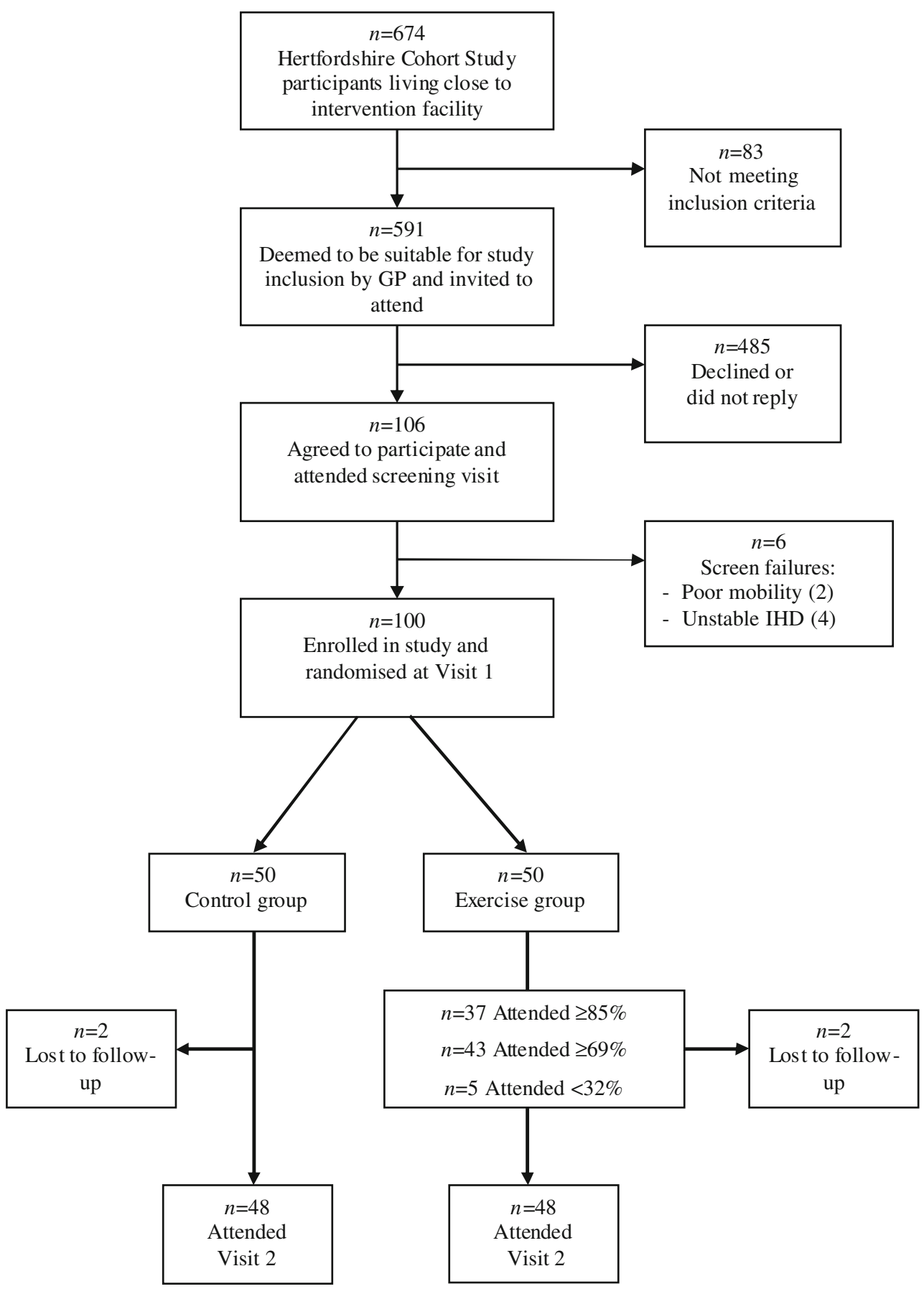

Secondary outcomes There were significant improvements in body weight, BMI and aerobic fitness in the exercise group compared with controls. However, there were no differences in lean mass, fat mass or percentage body fat measured by DEXA between the groups. Similarly blood pressure and lipid profiles did not change with exercise. There were no differences in fasting glucose, surrogate measures of insulin sensitivity or $\mathrm{HbA}_{1 \mathrm{c}}$ between the groups at follow-up, although a nonsignificant trend towards reduced $2 \mathrm{~h}$ glucose was observed in exercisers, with significantly lower insulin and C-peptide levels during the OGTT (Electronic supplementary material [ESM] Fig. 1a-c, ESM Table 1). The exercise group had a $68 \%$ reduction in the odds of abnormal glucose metabolism compared with controls (OR 0.32 [95\% CI 0.11-0.92] $p=0.035$ ). All of these results were similar in per protocol analyses comparing exercise attenders $(n=37)$ with controls, except that the reduction in $2 \mathrm{~h}$ glucose was statistically significant in this group ( $p=0.02$ unadjusted). 
Fig. 2 Forest plot of standardised trial outcomes. Results displayed are standardised difcontrol groups at follow-up (derived from the mean and standard deviation for each variable), adjusted for baseline values. *Components of zMS that have been standardised from the larger Hertfordshire Cohort Study ferences between exercise and

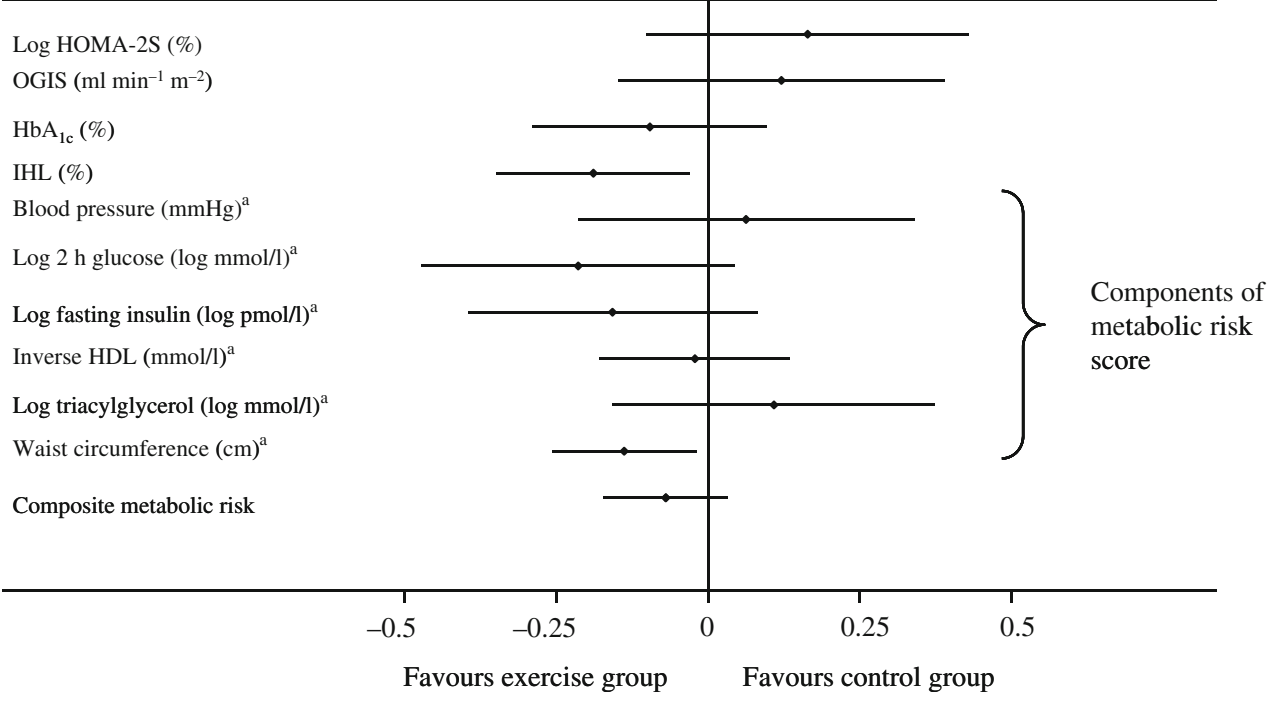

Differences in scores for the eight components of selfperceived functional status measured using the SF-8 are shown in ESM Table 2. In an intention-to-treat analysis, the only domain that improved significantly was social functioning. In a per protocol analysis adjusted for age and sex, there were significant improvements in role-physical, general health, social functioning and role-emotional domain scores. Summary physical and mental health scores remained unchanged after exercise in all analyses. No significant changes were noted in reported medication use between visits. A statistically significant reduction in the reported number of light meals per day was observed in the control group ( $p=0.005)$, but not in the frequency of snacks or main meals.

A subgroup of 62 study participants met the inclusion criteria and agreed to undergo magnetic resonance imaging and spectroscopy, having technically adequate scans performed at both visits. Scanned participants were more likely to be men than unscanned ones (58\% vs $53 \%$ ), to have lower BMI $\left(26.2 \mathrm{vs} 28.4 \mathrm{~kg} / \mathrm{m}^{2}\right)$ and to be of similar age (71.4 years). There were no changes in visceral or subcutaneous adipose tissue, or total abdominal fat crosssectional areas at follow-up between groups (data not shown). However, IHL was significantly reduced in the exercise group at follow-up (Table 1). These findings were similar in per protocol analyses.

\section{Discussion}

In this randomised controlled trial of 12 weeks of supervised aerobic exercise, the anticipated changes in the primary endpoint (zMS) and its constituents were not observed. However, there were significant improvements in other health-related anthropometric and metabolic measures, such as weight, BMI, waist circumference and IHL.
The risk of abnormal glucose metabolism at follow-up was reduced in exercisers. Insulin and C-peptide levels after glucose loading were lower after exercise, while glucose levels were equivalent, consistent with an improved insulin metabolism that may not have been apparent from the insulin sensitivity measures we used. Retention rates were high in both study groups, with three quarters of those randomised to exercise attending $85 \%$ or more of their prescribed sessions and being included in per protocol analyses, where findings were similar except that more domains of self-perceived health improved significantly, as did $2 \mathrm{~h}$ glucose levels.

This study suggests that, with appropriate supervision and clinical oversight, exercise interventions in healthy older people are safe. Compared with recent similar studies, our rate of adverse events was low [22, 23]. However, other studies have included higher risk groups such as those with established vascular disease. All of those invited to our study were deemed to be suitable for inclusion by their primary care physician. Of these, the $18 \%$ who agreed to participate had a detailed clinical assessment at their screening visit. Consequently, study participants were likely to be healthier than the age-matched general population from which they were recruited. Indeed, the negative zMSs at baseline in both groups confirm that study participants had healthier metabolic profiles than their peers in the Hertfordshire Cohort Study. Given that the benefits of aerobic exercise are already better established in less healthy individuals [24], the relatively good health of this cohort is one of the strengths of the study. In addition, all participants were of British Europid ethnic background, were born in Hertfordshire between 1931 and 1939, and were still residing there at the time of this study. While this limits the generalisability of the study to some extent, it may also limit the confounding effects of ethnic and cultural factors on the response to exercise, insofar as this 
is possible. A further factor limiting the generalisability of our findings is the relatively low recruitment rate of $18 \%$. One reason for this may be the rather intensive phenotypic characterisation (such as magnetic resonance imaging and muscle biopsy) that study participants were asked to undergo. In all likelihood, recruitment to a similar intervention with a lower volunteer measurement burden would be significantly higher. Thus, the feasibility of this type of intervention in a real-life setting remains to be determined.

It is conceivable that the duration of the intervention was too short or that the intensity of the exercise sessions, while rigorously maintained, was inadequate. However, over 12 weeks the intervention group lost weight, reduced their waist circumference and improved their fitness, and any concomitant improvements in metabolic risk might be expected to coincide with these changes, rather than taking additional weeks or months to become manifest. The nonsignificant changes seen in metabolic risk and insulin sensitivity were in the directions anticipated, suggesting that the effect size of the intervention on these outcomes may be smaller than we originally estimated. Exercise modality may be an important determinant of the degree of benefit obtained. While it has been suggested that resistance training confers increased metabolic benefits compared with aerobic exercise alone [25], recent work in a more contemporaneous cohort suggests aerobic interventions are more beneficial [23]. We sought to minimise heterogeneity in the exercise 'exposure' by using only cycle ergometers and closely monitoring and tailoring the intensity of exercise during each session. Additionally, the interval between the last exercise bout and the follow-up visit was consistently maintained (48 h) for each participant.

We did not include a dietary component in this intervention, as this could have introduced uncertainty relating to the mechanisms underlying any observed metabolic improvements. Furthermore, there is some evidence that more focused interventions with exercise alone achieve better results than combined exercise and dietary interventions [26], although many studies have found the converse to be true [27]. While all participants were asked not to change their diet, in practice the maintenance of consistent dietary intake is difficult to achieve and monitor in an ambulatory setting. We noted a statistically significant reduction in self-reported frequency of light meals per day in the control group. However, data on portion size and dietary composition were not recorded in this study, which is another limitation. It appears that dietary intake remained stable in the intervention group.

Our finding that aerobic exercise reduced IHL is novel and important, given that hepatic fat deposition is a major determinant of insulin sensitivity [28]. Animal studies suggest that exercise may have beneficial effects on liver steatosis in rats fed a high-fat diet [29]. To date, only observational studies in humans have suggested a beneficial effect of physical activity in this regard and good quality intervention studies are lacking [30]. Ours is the first study to demonstrate the efficacy of aerobic exercise in reducing liver fat in humans.

This study has demonstrated that 12 weeks of supervised aerobic exercise in appropriately screened, healthy older people confers important benefits in some, but not all, health-related outcomes.

Acknowledgements This study was funded by the Medical Research Council. F. M. Finucane was partly funded by a Graves Travelling Fellowship from the Irish Endocrine Society and a Bristol Myers Squibb/Sanofi Aventis Endocrine Research Bursary awarded by the Irish Committee on Higher Medical Training. S. J. Griffin receives support from the Department of Health NIHR Programme Grant funding scheme (RP-PG-0606-1259). We are grateful to the staff of the Wellcome Trust Clinical Research Facility in Addenbrooke's Hospital and the Field Epidemiology and Data Management Teams for assistance with study measures and data management, respectively. We would also like to thank $M$. Kershaw and the staff of Fitness Connections gymnasium in Hitchin, Hertfordshire, for accommodating the study. We are extremely grateful to the study participants for making this work possible.

Duality of interest The authors declare that there is no duality of interest associated with this manuscript.

\section{References}

1. Ekelund U, Franks PW, Sharp S, Brage S, Wareham NJ (2007) Increase in physical activity energy expenditure is associated with reduced metabolic risk independent of change in fatness and fitness. Diabetes Care 30:2101-2106

2. Laaksonen DE, Lakka HM, Lynch J et al (2003) Cardiorespiratory fitness and vigorous leisure-time physical activity modify the association of small size at birth with the metabolic syndrome. Diabetes Care 26:2156-2164

3. Paffenbarger RS Jr, Hyde RT, Wing AL, Lee IM, Jung DL, Kampert JB (1993) The association of changes in physicalactivity level and other lifestyle characteristics with mortality among men. N Engl J Med 328:538-545

4. Knowler WC, Barrett-Connor E, Fowler SE et al (2002) Reduction in the incidence of type 2 diabetes with lifestyle intervention or metformin. N Engl J Med 346:393-403

5. Lindstrom J, Ilanne-Parikka P, Peltonen M et al (2006) Sustained reduction in the incidence of type 2 diabetes by lifestyle intervention: follow-up of the Finnish Diabetes Prevention Study. Lancet 368:1673-1679

6. Clark AM, Hartling L, Vandermeer B, McAlister FA (2005) Metaanalysis: secondary prevention programs for patients with coronary artery disease. Ann Intern Med 143:659-672

7. Lavie CJ, Milani RV, Littman AB (1993) Benefits of cardiac rehabilitation and exercise training in secondary coronary prevention in the elderly. J Am Coll Cardiol 22:678-683

8. Donaldson L (2004) At least five a week: evidence on the impact of physical activity and its relationship to health. A report from the Chief Medical Officer. Department of Health, London

9. Graham I, Atar D, Borch-Johnsen K et al (2007) European guidelines on cardiovascular disease prevention in clinical practice: executive summary. Eur Heart J 28:2375-2414 
10. Crandall J, Schade D, Ma Y et al (2006) The influence of age on the effects of lifestyle modification and metformin in prevention of diabetes. J Gerontol A Biol Sci Med Sci 61:1075-1081

11. Lindstrom J, Peltonen M, Eriksson JG et al (2008) Determinants for the effectiveness of lifestyle intervention in the Finnish Diabetes Prevention Study. Diabetes Care 31:857-862

12. Finucane FM, Horton J, Purslow LR et al (2009) Randomised controlled trial of the efficacy of aerobic exercise in reducing metabolic risk in healthy older people: The Hertfordshire Physical Activity Trial. BMC Endocr Disord 9:15

13. Syddall HE, Aihie Sayer A, Dennison EM, Martin HJ, Barker DJ, Cooper C (2005) Cohort profile: the Hertfordshire cohort study. Int J Epidemiol 34:1234-1242

14. Salamone LM, Fuerst T, Visser M et al (2000) Measurement of fat mass using DEXA: a validation study in elderly adults. J Appl Physiol 89:345-352

15. Ware J, Kosinski M, Dewey J, Gandek B (2001) How to score and interpret single-item health status measures: a manual for users of the SF-8 Health Survey. QualityMetric, Boston

16. Matthews DR, Hosker JP, Rudenski AS, Naylor BA, Treacher DF, Turner RC (1985) Homeostasis model assessment: insulin resistance and beta-cell function from fasting plasma glucose and insulin concentrations in man. Diabetologia 28:412-419

17. Mari A, Pacini G, Murphy E, Ludvik B, Nolan JJ (2001) A model-based method for assessing insulin sensitivity from the oral glucose tolerance test. Diabetes care 24:539-548

18. Storer TW, Davis JA, Caiozzo VJ (1990) Accurate prediction of VO2max in cycle ergometry. Med Sci Sports Exerc 22:704712

19. Borg GA (1982) Psychophysical bases of perceived exertion. Med Sci Sports Exerc 14:377-381

20. Franks PW, Ekelund U, Brage S, Wong MY, Wareham NJ (2004) Does the association of habitual physical activity with the metabolic syndrome differ by level of cardiorespiratory fitness? Diabetes Care 27:1187-1193
21. White IR, Thompson SG (2005) Adjusting for partially missing baseline measurements in randomized trials. Stat Med 24:993-1007

22. McDermott MM, Ades P, Guralnik JM et al (2009) Treadmill exercise and resistance training in patients with peripheral arterial disease with and without intermittent claudication: a randomized controlled trial. JAMA 301:165-174

23. Davidson LE, Hudson R, Kilpatrick K et al (2009) Effects of exercise modality on insulin resistance and functional limitation in older adults: a randomized controlled trial. Arch Intern Med 169:122-131

24. Jolliffe JA, Rees K, Taylor RS, Thompson D, Oldridge N, Ebrahim S (2001) Exercise-based rehabilitation for coronary heart disease. Cochrane Database Syst Rev, Issue 1, Art no.: CD001800. doi:10.1002/14651858.CD001800

25. Cuff DJ, Meneilly GS, Martin A, Ignaszewski A, Tildesley HD, Frohlich JJ (2003) Effective exercise modality to reduce insulin resistance in women with type 2 diabetes. Diabetes Care 26:2977-2982

26. Lambert CP, Wright NR, Finck BN, Villareal DT (2008) Exercise but not diet-induced weight loss decreases skeletal muscle inflammatory gene expression in frail obese elderly persons. J Appl Physiol 105:473-478

27. Stefanick ML, Mackey S, Sheehan M, Ellsworth N, Haskell WL, Wood PD (1998) Effects of diet and exercise in men and postmenopausal women with low levels of HDL cholesterol and high levels of LDL cholesterol. N Engl J Med 339:12-20

28. Marchesini G, Brizi M, Morselli-Labate AM et al (1999) Association of nonalcoholic fatty liver disease with insulin resistance. Am J Med 107:450-455

29. Gauthier MS, Couturier K, Charbonneau A, Lavoie JM (2004) Effects of introducing physical training in the course of a 16-week high-fat diet regimen on hepatic steatosis, adipose tissue fat accumulation, and plasma lipid profile. Int J Obes Relat Metab Disord 28:1064-1071

30. Spassiani NA, Kuk JL (2008) Exercise and the fatty liver. Appl Physiol Nutr Metab 33:802-807 